\title{
Retraction
}

\section{Retracted: Chronic Kidney Disease-Effect of Oxidative Stress}

\author{
Chinese Journal of Biology
}

Received 21 August 2019; Accepted 21 August 2019; Published 9 December 2019

Copyright (c) 2019 Chinese Journal of Biology. This is an open access article distributed under the Creative Commons Attribution License, which permits unrestricted use, distribution, and reproduction in any medium, provided the original work is properly cited.

Chinese Journal of Biology has retracted the article titled "Chronic Kidney Disease-Effect of Oxidative Stress" [1]. The article was found to contain a substantial amount of material from the following published articles, including the following sources cited as $[1,15,16,7]$ :

(i) M. P. Kao, D. S. Ang, A. Pall, and A. D. Struthers, "Oxidative stress in renal dysfunction: mechanisms, clinical sequelae and therapeutic options," Journal of Human Hypertension, vol. 24, no. 1, pp. 1-8, 2010, https://doi.org/10.1038/jhh.2009.70 [2].

(ii) E. O. Gosmanova and N.-A. Le, "Cardiovascular complications in CKD patients: role of oxidative stress," Cardiology Research and Practice, vol. 2011, Article ID 156326, 8 pages, 2011, https://doi.org/10. 4061/2011/156326 [3].

(iii) K.-L. Kuo and D.-C. Tarng, "Oxidative stress in chronic kidney disease," Adaptive Medicine, vol. 2, no. 2, pp. 87-94, 2010, DOI: 10.4247/AM.2010.ABA010, http://www.sastam.org.tw/adaptive_medicine/2010-2. aspx [4].

(iv) A. Popolo, G. Autore, A. Pinto and S. Marzocco, Oxidative stress in patients with cardiovascular disease and chronic renal failure," Free Radical Research, vol. 47, no. 5, pp. 346-356, 2013, DOI: 10.3109/10715762.2013.779373 [5] (not cited).

(v) V. Krane, and C. Wanner, "The metabolic burden of diabetes and dyslipidaemia in chronic kidney disease, Nephrology Dialysis Transplantation," vol. 17, no. suppl_11, 2002, pp. 23-27, https://doi.org/10. 1093/ndt/17.suppl_11.23 [6].

\section{References}

[1] S. P. Meenakshi Sundaram, S. Nagarajan, and A. J. Manjula Devi, "Chronic kidney disease-effect of oxidative stress," Chinese Journal of Biology, vol. 2014, Article ID 216210, 6 pages, 2014.

[2] M. P. C. Kao, D. S. C. Ang, A. Pall, and A. D. Struthers, "Oxidative stress in renal dysfunction: mechanisms, clinical sequelae and therapeutic options," Journal of Human Hypertension, vol. 24, no. 1, pp. 1-8, 2010.

[3] E. O. Gosmanova and N.-A. Le, "Cardiovascular complications in CKD patients: role of oxidative stress," Cardiology Research and Practice, vol. 2011, Article ID 156326, 8 pages, 2011.

[4] K.-L. Kuo and D.-C. Tarng, "Oxidative stress in chronic kidney disease," Adaptive Medicine, vol. 2, no. 2, pp. 87-94, 2010.

[5] A. Popolo, G. Autore, A. Pinto, and S. Marzocco, "Oxidative stress in patients with cardiovascular disease and chronic renal failure," Free Radical Research, vol. 47, no. 5, pp. 346-356, 2013, (not cited).

[6] V. Krane and C. Wanner, "The metabolic burden of diabetes and dyslipidaemia in chronic kidney disease," Nephrology Dialysis Transplantation, vol. 17, no. suppl_11, pp. 23-27, 2002. 


\title{
Chronic Kidney Disease-Effect of Oxidative Stress
}

\author{
Subha Palaneeswari Meenakshi Sundaram, ${ }^{1}$ \\ Sivakumar Nagarajan, ${ }^{2}$ and Arcot Jagdeeshwaran Manjula Devi ${ }^{1}$ \\ ${ }^{1}$ Department of Biochemistry, Sree Balaji Medical College and Hospital, Bharath University, \\ CLC Works Road, Chrompet, Chennai 600 044, India \\ ${ }^{2}$ Department of Nephrology, Sree Balaji Medical College and Hospital, Bharath University, \\ CLC Works Road, Chrompet, Chennai 600 044, India
}

Correspondence should be addressed to Subha Palaneeswari Meenakshi Sundaram; msubhavish@gmail.com

Received 27 September 2013; Accepted 30 October 2013; Published 21 January 2014

Academic Editors: A. Castañeyra-Perdomo, W. Ding, and R. Malli

Copyright (C) 2014 Subha Palaneeswari Meenakshi Sundaram et al. This is an open access article distributed under the Creative Commons Attribution License, which permits unrestricted use, distribution, and reproduction in any medium, provided the original work is properly cited.

Chronic kidney disease (CKD) is a growing health problem with increasing incidence. The annual mortality of end-stage renal disease patients is about $9 \%$, which is $10-20$ fold higher than the general population, approximately $50 \%$ of these deaths are due to cardiovascular (CV) disease. CV risk factors, such as diabetes, hypertension, and hyperlipidemia, are strongly associated with poor outcome. Many other nontraditional risk factors such as inflammation, infection, oxidative stress, anemia, and malnutrition are also present. In this review we will focus on the role of oxidative stress in chronic kidney disease.

\section{Introduction}

Chronic kidney disease (CKD) is a growing health problem with increasing incidence. In the United States, approximately 8 million adults have stage 3 CKD and about 400000 have end-stage renal disease (ESRD), with over 300000 of them on maintenance dialysis (MHD). The annual mortality of ESRD patients is about $9 \%$, which is $10-20$-fold higher than the general population; approximately $50 \%$ of these deaths are due to cardiovascular (CV) disease [1]. CKD is associated with high cardiovascular risk which is not fully due to traditional CV risk factors. CV risk factors, such as diabetes, hypertension, and hyperlipidemia, are strongly associated with poor outcome; other nontraditional risk factors are also present such as renal-specific risk factors: uraemia or dialysis and extrarenal risk factors: proteinuria, chronic volume overload, increased renin-angiotensin system activity, inflammation, infection, oxidative stress, altered calcium and phosphorus metabolism, anemia, malnutrition, elevated levels of homocysteine, uraemic toxins, and thrombogenic factors [1]. Changes in serum cytokines begin at an early phase of renal dysfunction. High levels of cytokines lead to changes in the levels of many acute phase proteins like creactive protein (CRP), albumin, brain natriuretic peptide (BNP), adiponectin, fetuin, and asymmetric dimethylargainine. Changes in these proteins can predict intima-media thickness of carotid artery and left ventricular hypertrophy leading to accelerated cardiovascular mortality. Chronic inflammation is a possible mediator between microvascular and macrovascular disease. Oxidative stress may accelerate atherogenesis, and hence make a significant contribution to the increased CV disease burden in CKD patients. Oxidative stress may actually represent a new target for therapeutic intervention. In this review, first we will discuss the role and the biochemical burden of diabetes and dyslipidemia in CKD and then focus on the role of oxidative stress in CKD.

\section{Dyslipidemia and CKD}

Dyslipidemia is related to the incidence and prognosis of CKD through mechanisms such as inflammation and increased oxidative stress, which could cause endothelial damage and atherosclerosis [2]. Studies have reported that 
obesity and metabolic syndrome adversely affects renal function and may be associated with morbidity and mortality in patients with CKD [3-5]. The expression of inflammatory genes and genes implicated in insulin resistance is increased in the glomeruli of patients with obesity-related nephropathy. Adipose tissue is the source of active substances known as adipokines. Many adipokines including IL-6, TNF- $\alpha$, and plasminogen activator inhibitor-1 (PAI-1) may cause tissue damage by a direct proinflammatory mechanism or insulin resistance and can lead to inflammatory changes in the kidney. The pattern of dyslipidemia in CKD differs from the non-CKD [6] and is similar to those found in dyslipidemia associated with diabetes [7]. The most important abnormalities are an increase in serum triglyceride (TGL), predominantly very low-density lipoprotein (VLDL), and intermediate-density lipoprotein (IDL), mainly due to abnormal lipase function [6] causing hypertriglyceridemia and low high-density lipoprotein (HDL). Even though the total cholesterol level may be within normal range, the lipid profile pattern may be abnormal with predominantly the small dense LDL particles which are atherogenic; Lipoprotein-a (Lp-a) component is also elevated. The Chronic Renal Impairemnt in Birmingham (CRIB) study has confirmed the presence of dyslipidemia in early CKD. The components of TGL-rich lipoproteins, such as apolipoprotein B, apolipoprotein C-III, and apolipoprotein $\mathrm{E}$ are elevated in ESRD [7]. In patients with hypertriglyceridemia, diabetic nephropathy and ESRD on hemodialysis (HD) small dense LDL preferentially accumulate, thus leading to increased risk of peripheral vascular disease, coronary artery disease, and myocardial infarction [8].

\section{Diabetes and CKD}

Hyperglycemia leads to vascular damage via several mechanisms. The four main mechanisms are increased flux through the hexosamine path, increased flux through polyol pathway, activation of protein kinase $\mathrm{C}$ (PKC), and increased formation of advanced glycation end-products (AGE). Hyperglycemia leads to activation of hexosamine pathway, which inturn leads to changes in gene expression, protein function, and transcription of PAI-1, thus leading to the pathogenesis of diabetic complications [7]. The polyol pathway is implicated in diabetic neuropathy and diabetic retinopathy. Cellular hyperglycemia leads to PKC activation which inturn affects the expression and function of endothelial nitric oxide synthase, endothelin-1, vascular endothelial growth factor, transforming growth factor-beta, nuclear factor- $\kappa \mathrm{B}$, and nicotine adenine diphosphate oxidase [9].

The effects of AGEs can be classified as receptorindependent or receptor-dependent and can act intracellularly or on cell surface receptors such as the receptor for AGEs (RAGEs). Advanced glycation occurs over a prolonged period of time and mainly affects the long-lived proteins such as connective tissue matrix; in particular, basement membrane components such as type-IV collagen are prime targets, including other long-lived proteins such as myelin, tubulin, plasminogen activator 1 , and fibrinogen $[10,11]$.
Glycation leads to structural alterations, increased stiffness, and resistance to proteolytic digestion. AGEs may promote atherosclerosis by their interaction with AGE receptors on the endothelial cells, causing the expression of adhesion molecules and attraction of monocytes into the vessel wall. AGEs also alter the plasma proteins, thus inducing the expression of proinflammatory and procoagulatory molecules by the endothelial cells, for example, thrombomodulin, expression of cytokines, and growth factors by macrophages and mesangial cells, for example, IL-1, IL-2, IL-6, and tumor necrosis factor- $\alpha$. These changes result in inflammation, oxidative stress, and coagulopathy and promote atherosclerosis [9]. In CKD there is an accumulation of AGEs, which may be due to increased concentration of reactive dicarbonyls in uremia and decreased renal clearance of AGEs. Elevated serum levels of neopterin (monocyte activation marker) and pentosidine correlate with the rate of progression of diabetic nephropathy [11].

The receptor-dependent effects of AGE are mediated through its interactions with various proteins like the RAGE protein, AGE receptors, and the ezrin, radixin, and moesin (ERM) family. RAGE is a member of the immunoglobulin superfamily of receptors. AGEs through their interaction with RAGE activate secondary messenger pathways such as protein kinase $C$. The key target of RAGE signaling is nuclear factor- $\kappa \mathrm{B}(\mathrm{NF}-\mathrm{kB})$, which increases transcription of many proteins, including intercellular adhesion molecule1. The strongest evidence for AGEs in the development of diabetic nephropathy has come from studies targeting the AGE-RAGE pathway $[11,12]$.

\section{Oxidative Stress and Inflammation in CKD}

Oxidative stress is a state of imbalance between free radicals production and its degradation by antioxidant systems with increased accumulation of the radicals. Over $90 \%$ of reactive oxygen species (ROS) formation occurs in mitochondria during metabolism of oxygen when some electrons passing the electron transport chain may leak from the main path and can directly reduce oxygen molecules to the superoxide anion $[1,11,13]$. ROS could also be deliberately synthesized in phagocytic cells, as well as in vascular wall and various other tissues by enzymes such as $\mathrm{NAD}[\mathrm{P}] \mathrm{H}$ oxidase, myeloperoxidase (MPO), xanthine oxidase, cyclooxygenase, and lipoxygenase. The release of an ROS may lead to the production of several through radical chain reactions. These potentially deleterious reactions are regulated by enzymatic and nonenzymatic antioxidants which eliminate prooxidants and scavenge-free radicals. Superoxide dismutase (SOD), glutathione peroxidase, glutathione reductase, and catalase are among the main enzymatic antioxidants. Glutathione, thiols, ascorbic acid, alpha-tocopherol (vitamin E), mixed carotenoids, and bioflavonoids are the major nonenzymatic antioxidant defense processes [11].

At low concentrations, ROS like superoxide anion, hydrogen peroxide, hydroxyl radical, hypochlorite ion, and hydroperoxyl radical play vital role in many physiologic functions. For example, ROS are known regulators of nitric 


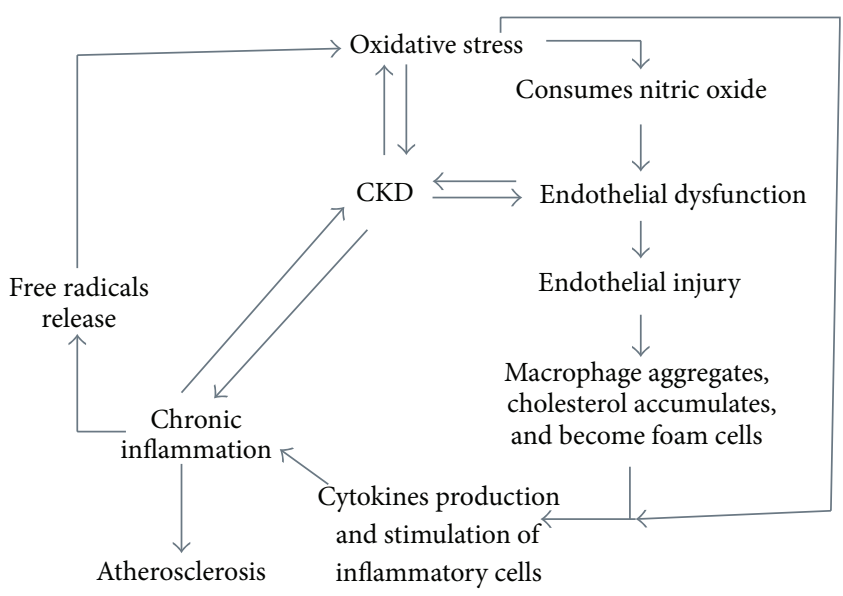

FIGURE 1: Role of oxidative stress in chronic inflammation and CKD.

oxide synthesis, intracellular signaling cascades, and are also involved in the modulation of immune responses, apoptosis, and mutagenesis. ROS produced during phagocytic burst is a key defense mechanism against environmental pathogens. They also lead to the formation of peroxynitrite, which is cytotoxic and also responsible for both increased platelet aggregation and vasoconstriction. When excess ROS are produced they can react with various biomolecules, thus altering their structure and function, resulting in cellular damage and pathologic processes, including atherosclerosis [13]. Together with proinflammatory cytokines, angiotensin II, and advanced glycation end-products, ROS release will augment the ROS generation process (Figure 1).

4.1. Biomarkers of Oxidative Stress. Free radicals have a very short half-life (in seconds); hence their evaluation in clinical setting is difficult. But there are more stable marker molecules with longer half-lives, ranging from hours to weeks, which can be used to assess oxidative stress associated with diverse diseases, including uremia [1]. Some of these are oxidation byproducts, such as oxidized low-density lipoprotein (LDL), reactive carbonyl compounds, advanced glycosylation endproduct (AGE), and oxidized thiol compounds, which can potentially contribute to the pathogenesis of $\mathrm{CV}$ disease, inflammation, and other complications of uremia $[12,14,15]$.

4.1.1. Circulating Biomarkers of Oxidative Stress in Chronic Kidney Disease Patients [16]

\section{Carbohydrates}

Advanced glycosylation end-products (AGEs)

Reactive aldehydes

\section{Proteins}

Amine oxidation

Advanced oxidation protein products (AOPPs)

Carbonyl formation

Thiol oxidation

\section{Amino Acids}

Cysteine

Homocysteine

Isoaspartate

Nitrotyrosine

Lipids

Advanced lipoxidation end-products (ALEs)

Exhaled alkanes

Malondialdehyde (MDA)

Lipid hydroperoxides

Oxidized low-density lipoprotein (ox-LDL)

Arachidonic Acid Derivatives

F2 isoprostanes

Isolevuglandins

Nucleic Acids

8-Hydroxy $2^{\prime}$-deoxyguanosine (8-OHdG).

4.2. Role of Oxidative Stress in Renal Dysfunction. Many clinical studies have confirmed the major role played by oxidative stress in renal dysfunction. In dialysis patients, AGEs have been associated with atherosclerosis and dialysis-associated amyloidosis. Malondialdehyde (MDA) has been suggested as one of the best predictors of cardiovascular disease in hemodialysis patients [16, 17]. 8-Hydroxydeoxyguanosine (8-OHdG) concentrations were highest in long-term HD patients. Witko-Sarsat et al. identified the presence of advanced oxidation protein products (AOPPs) in the plasma of uremic patients, and the AOPP levels increased as renal function declined [18]. Drüeke et al. found that levels of AOPP strongly correlated with carotid artery intima-medial thickness (IMT) in patients with ESRD [19]. A similar finding was reported by Yang et al. in CKD [20]. Additionally, significant positive correlation between carotid artery IMT and thiobarbituric acid reactive substances (TBARS) and MPO and negative correlation between carotid artery IMT and reduced SOD and plasma sulfhydryl were reported in patients with ESRD. Shoji et al. [21] observed a statistical trend in correlation between carotid artery IMT and autoantibodies against oxidatively modified LDL (oxLDL). A stronger correlation was observed between femoral artery IMT and autoantibodies against oxLDL in ESRD $[15,22]$. In CKD patients not on maintenance dialysis, autoantibodies against oxLDL correlated with carotid atherosclerosis and strongly predicted cardiovascular mortality during 4 years of follow-up study in hemodialysis patients [15].

4.3. Mechanisms That Lead to Increased Oxidative Stress in $C K D$. Different mechanisms could explain the increased oxidative stress in CKD. Basic characteristics of renal patients such as advanced age, diabetes, renal hypertension, lower levels of antioxidant vitamin due to dietary restriction of fresh 
fruits and vegetables (to avoid hyperkalaemia), and failure of ROS clearance with decline in renal function are implicated in increased oxidative stress and progression of CKD; and oxidative stress seems to happen early during the course of renal decline $[1,16,22]$. The pathogenesis of oxidative stress in CKD patients is complex and includes uremia-related factors and dialysis-related factors.

4.3.1. Uremia-Related Factors. Studies have shown that the resting serum levels of superoxide anion were higher in HD patients than in healthy controls. Several evidence suggests that antioxidant enzymes are altered as renal function declines and are profoundly impaired in patients with uremia. Investigators have also found that $\mathrm{CKD}$ was associated with low concentration of serum selenium and lower platelet glutathione peroxidase (GPx) activity [16]. Ceballos-Picot et al. [23] demonstrated lower serum levels of glutathione and plasma GPx activity in renal failure patients. CKD is also associated with a profound disturbance in nitric oxide system, as it increases the concentration of endogenous NOS inhibitors.

4.3.2. Dialysis-Related Factors. The bioincompatibility of HD systems plays a pivotal role in ROS production by activation of polymorphonuclear neutrophils. In particular, two major components of the HD system contribute to oxidative stress: the dialyzer membrane and the trace amount of endotoxin in the dialysate. There may be a concomitant loss of antioxidant vitamins by dialysis process $[1,16]$. Plasma vitamin $\mathrm{E}$ and selenium are reduced; there is also significant deficiency and downregulation of glutathione peroxidase, copper-zinc superoxide dismutase, and manganese superoxide dismutase in renal dysfunction [16].

4.3.3. Role of Inflammation in CKD. CKD is a low grade inflammatory process in itself. Chronic inflammation can itself lead to increased oxidative stress in advanced renal disease, with malnutrition, chronic volume overload, and autonomic dysfunction as some factors implicated in the increased inflammatory state seen in renal impairment. Studies have observed association between many mediators of inflammation such as CRP, IL-6, TNF-a, fibrinogen, and renal dysfunction. In CKD patients, the polymorphonuclear neutrophils are activated and MPO is generated, triggering ROS activation [1].

4.4. Clinical Effect of Oxidative Stress in CKD. The important consequence of oxidative stress in CKD is endothelial dysfunction and the progression of other complications like atherosclerosis, anemia, and amyloidosis. Several qualitative LDL changes like increased carbamylation, AGE products formation, and oxidative stress seen in dialysis patients result in endothelial dysfunction, platelet aggregation, metaloproteinase expression, and thrombogenesis. Studies show that in CKD the LDL is more susceptible to in vitro oxidation than in the healthy [24].

In CKD, depending on the severity, the prevalence of left ventricular hypertrophy (LVH) varies between 40 to $75 \%$.
The aetiology of LVH in CKD is multifactorial such as volume loading conditions (as in dialysis-dependent patients), hypertension, and oxidative stress. In vivo studies have shown that oxidative stress induces several cardiomyocyte hypertrophy signalling kinases (GTP-binding protein Ras, mitogen activated protein kinase, protein kinase $C$, tyrosine kinase Src, Jun-nuclear kinase, and transcription factors $(\kappa \mathrm{B}$, activator-protein-1, Ets), which stimulate matrix metalloproteinases expression [25]. ROS also activate apoptosis signalling kinase- 1 and nuclear factor $\kappa \mathrm{B}$, which are implicated in cardiomyocyte hypertrophy.

4.4.1. Amyloidosis. In the presence of oxidative stress, ROS can directly modify the function of proteins through the formation of oxidized amino acids. Miyata et al. demonstrated that AGEs occur in beta2-microglobulin deposits of longterm HD patients, suggesting that oxidative stress promotes amyloidosis due to protein denaturation $[16,26]$.

4.4.2. Anemia. Previous studies indicated that the serum of HD patients has higher levels of MDA in erythrocytes and a severe deficiency in vitamin content, which may shorten the lifespan of erythrocytes in CKD. Antioxidant therapy improved anemia in CKD and reduced the requirement for erythropoiesis-stimulating agents (ESAs) in them $[1,27]$.

\section{Role of Antioxidant}

Many studies have examined the effect of antioxidant interventions on oxidative stress markers in CKD. The SPACE (Secondary Prevention with Antioxidants of Cardiovascular Disease in End-Stage Renal Disease) study observed a reduction in the primary composite outcome, consisting of myocardial infarction, ischemic stroke, peripheral vascular disease, and unstable angina in ESRD patients receiving vitamin E [28]. Statins also reduce inflammatory markers. A decrease in cumulative incidence for stroke, total coronary events, total cardiovascular events, and allcause mortality was observed in the Anglo-Scandinavian Cardiac Outcomes Trial-Lipid Lowering Arm (ASCOTLLA) $[15,29]$ study which also included a subgroup of CKD patients. Wassmann et al. have described a relationship between atorvastatin-induced improvement of endothelial dysfunction and reduced free-radical release in the vasculature associated with a statin-induced downregulation of angionten-I-receptor expression [7, 30]. Statins can also inhibit the activation and proliferation of lymphocytes and smooth-muscle cells, as well as chemotaxis and oxidativeburst of macrophages; platelet adhesion and aggregation are also blocked. The Study of Heart and Renal Protection (SHARP) tells the cardiovascular benefits of statin use in CKD patients [15, 31].

Antioxidant-based dialysis membranes, such as vitamin E-modified cellulose membranes, have been shown to improve endothelial dysfunction and increase oxidised LDL. Recently, there have been promising reports of haemolipodialysis using vitamin $\mathrm{C}$ and vitamin E-containing liposomes in the dialysate to reduce dialysis-induced oxidative stress; 
the liposomes get oxidised and killed because of their high degree of unsaturation and thus act as the "radical sink" $[1,32]$.

Angiotensin-converting enzyme inhibitors and angiotensin receptor blockers are highly effective inhibitors of angiotensin II-dependent NADPH oxidase activation and have been used successfully in the clinical setting, especially in patients with CKD/ESRD.

\section{Conclusion}

CKD patients are affected by multiple concomitant conditions like diabetes, dyslipidemia, and hypertension which are all associated with oxidative stress. The presence of CKD appears to further enhance the oxidative stress independently from the underlying conditions. Hemodialysis has also been demonstrated to contribute to the oxidative stress. Increasing evidence suggests that oxidative stress is a plausible independent cardiovascular risk factor in CKD. Cardiovascular disease is an important cause of morbidity and mortality in patients with impaired kidney function. Furthermore, identification of biochemical and/or functional biomarkers that could be used in clinical practice to monitor oxidative imbalance in CKD may allow the development of an optimal intervention strategy to reduce oxidative stress in CKD.

\section{Conflict of Interests}

The authors declare that there is no conflict of interests regarding the publication of this paper.

\section{References}

[1] M. P. Kao, D. S. Ang, A. Pall, and A. D. Struthers, "Oxidative stress in renal dysfunction: mechanisms, clinical sequelae and therapeutic options," Journal of human hypertension, vol. 24, no. 1, pp. 1-8, 2010.

[2] W. H. Sheu and Y. Sheen, "Metabolic syndrome and renal injury," Cardiology Research and Practice, vol. 1, no. 1, Article ID 567389, 2011.

[3] P. Iglesias and J. J. Díez, "Adipose tissue in renal disease: clinical significance and prognostic implications," Nephrology Dialysis Transplantation, vol. 25, no. 7, pp. 2066-2077, 2010.

[4] I. Lee, C. Huang, W. Lee, H. Lee, and W. H.-H. Sheu, "Aggravation of albuminuria by metabolic syndrome in type 2 diabetic Asian subjects," Diabetes Research and Clinical Practice, vol. 81, no. 3, pp. 345-350, 2008.

[5] J. Lucove, S. Vupputuri, G. Heiss, K. North, and M. Russell, "Metabolic syndrome and the development of CKD in American Indians: the StrongHeart Study," American Journal of Kidney Diseases, vol. 51, no. 1, pp. 21-28, 2008.

[6] M. P. Delaney, C. P. Price, and D. J. Newman, "Edmund lamb. Kidney disase," in Tietz Texstbook of Clinical Biochemistry, pp. 1670-1745, Saunders, 4th edition, 2006.

[7] V. Krane and C. Wanner, "The metabolic burden of diabetes and dyslipidaemia in chronic kidney disease," Nephrology Dialysis Transplantation, vol. 17, no. 11, pp. 23-27, 2002.

[8] T. Quaschning, V. Krane, T. Metzger, and C. Wanner, "Abnormalities in uremic lipoprotein metabolism and its impact on cardiovascular disease," American Journal of Kidney Diseases, vol. 38, supplement 1, pp. S14-S19, 2001.

[9] H. J. Goldberg, J. Scholey, and I. G. Fantus, "Glucosamine activates the plasminogen activator inhibitor 1 gene promoter through Sp1 DNA binding sites in glomerular mesangial cells," Diabetes, vol. 49, no. 5, pp. 863-871, 2000.

[10] M. Brownlee, "Biochemistry and molecular cell biology of diabetic complications," Nature, vol. 414, no. 6865, pp. 813-820, 2001.

[11] S. Goh and M. E. Cooper, "The role of advanced glycation end products in progression and complications of diabetes," Journal of Clinical Endocrinology and Metabolism, vol. 93, no. 4, pp. 1143-1152, 2008.

[12] A. Flyvbjerg, L. Denner, B. F. Schrijvers et al., "Long-term renal effects of a neutralizing RAGE antibody in obese type 2 diabetic mice," Diabetes, vol. 53, no. 1, pp. 166-172, 2004.

[13] J. M. Forbes, T. Soulis, V. Thallas et al., "Renoprotective effects of a novel inhibitor of advanced glycation," Diabetologia, vol. 44, no. 1, pp. 108-114, 2001.

[14] H. Honda, M. Ueda, S. Kojima et al., "Assessment of myeloperoxidase and oxidative $\alpha$-antitrypsin in patients on hemodialysis," Clinical Journal of the American Society of Nephrology, vol. 4, no. 1, pp. 142-151, 2009.

[15] N. Le and E. O. Gosmanova, "Cardiovascular complications in CKD patients: role of oxidative stress," Cardiology Research and Practice, vol. 1, no. 1, Article ID 156326, 2011.

[16] K.-L. Kuo and D.-C. Tarng, "Oxidative stress in chronic kidney disease," Adaptive Medicine, vol. 2, no. 2, pp. 87-94, 2010.

[17] W. Dröge, "Free radicals in the physiological control of cell function," Physiological Reviews, vol. 82, no. 1, pp. 47-95, 2002.

[18] V. Witko-Sarsat, M. Friedlander, T. N. Khoa et al., "Advanced oxidation protein products as novel mediators of inflammation and monocyte activation in chronic renal failure," Journal of Immunology, vol. 161, no. 5, pp. 2524-2532, 1998.

[19] T. Drüeke, V. Witko-Sarsat, Z. Massy et al., "Iron therapy, advanced oxidation protein products, and carotid artery intima-media thickness in end-stage renal disease," Circulation, vol. 106, no. 17, pp. 2212-2217, 2002.

[20] X. Yang, F. Hou, Q. Wu et al., "Increased levels of advanced oxidation protein products are associated with atherosclerosis in chronic kidney disease," Zhonghua Nei Ke Za Zhi, vol. 44, no. 5, pp. 342-346, 2005.

[21] T. Shoji, E. Kimoto, K. Shinohara et al., "The association of antibodies againstoxidized low-density lipoprotein with atherosclerosis in hemodialysis patients," Kidney International, Supplement, vol. 63, no. 84, pp. S128-S130, 2003.

[22] F. Locatelli, B. Canaud, K. Eckardt, P. Stenvinkel, C. Wanner, and C. Zoccali, "Oxidative stress in end-stage renal disease: an emerging treat to patient outcome," Nephrology Dialysis Transplantation, vol. 18, no. 7, pp. 1272-1280, 2003.

[23] I. Ceballos-Picot, V. Witko-Sarsat, M. Merad-Boudia et al., "Glutathione antioxidant system as a marker of oxidative stress in chronic renal failure," Free Radical Biology and Medicine, vol. 21, no. 6, pp. 845-853, 1996.

[24] R. J. Esper, R. A. Nordaby, J. O. Vilariño, A. Paragano, J. L. Cacharrón, and R. A. Machado, "Endothelial dysfunction: a comprehensive appraisal," Cardiovascular Diabetology, vol. 5, article 4, 2006.

[25] E. Paoletti, D. Bellino, P. Cassottana, D. Rolla, and G. Cannella, "Left ventricular hypertrophy in nondiabetic predialysis CKD," American Journal of Kidney Diseases, vol. 46, no. 2, pp. 320-327, 2005. 
[26] T. Miyata, C. V. de Strihou, K. Kurokawa, and J. W. Baynes, "Alterations in nonenzymatic biochemistry in uremia: origin and significance of "carbonyl stress" in long-term uremic complications," Kidney International, vol. 55, no. 2, pp. 389-399, 1999.

[27] V. Lahera, M. Goicoechea, S. G. de Vinuesa et al., "Oxidative stress in uremia: the role of anemia correction," Journal of the American Society of Nephrology, vol. 17, pp. S174-S177, 2006.

[28] M. Boaz, S. Smetana, T. Weinstein et al., "Secondary prevention with antioxidants of cardiovascular disease in endstage renal disease (SPACE): randomised placebo-controlled trial," The Lancet, vol. 356, no. 9237, pp. 1213-1218, 2000.

[29] P. S. Sever, B. Dahlöf, N. R. Poulter et al., "Prevention of coronary and stroke events with atorvastatin in hypertensive patients who have average or lower-than-average cholesterol concentrations, in the Anglo-Scandinavian Cardiac Outcomes Trial-Lipid Lowering Arm (ASCOT-LLA): a multicentre randomised controlled trial," The Lancet, vol. 361, no. 9364, pp. 1149-1158, 2003.

[30] S. Wassmann, U. Laufs, A. T. Bäumer et al., "HMG-CoA reductase inhibitors improve endothelial dysfunction in normocholesterolemic hypertension via reduced production of reactive oxygen species," Hypertension, vol. 37, no. 6, pp. $1450-$ 1457, 2001.

[31] SHARP Collaborative Group, "Study of Heart and Renal Protection (SHARP): randomized trial to assess the effects of lowering low-density lipoprotein cholesterol among 9, 438 patients with chronic kidney disease," American Heart Journal, vol. 160, no. 5, pp. 785-794, 2010.

[32] M. L. Wratten, C. Navino, C. Tetta, and G. Verzetti, "Haemolipodialysis," Blood Purification, vol. 17, no. 2-3, pp. 127-133, 1999. 